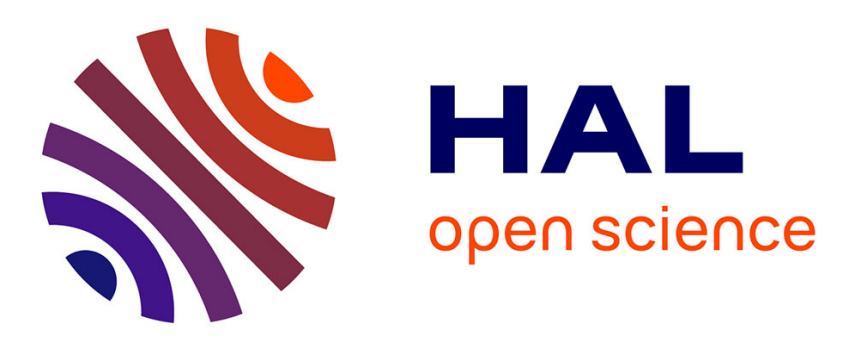

\title{
La France et l'Europe centrale à Versailles : objectifs stratégiques et combinaisons diplomatiques
}

Frédéric Dessberg

\section{To cite this version:}

Frédéric Dessberg. La France et l'Europe centrale à Versailles : objectifs stratégiques et combinaisons diplomatiques. Guerres mondiales et conflits contemporains, 2021, 282 (2), pp.15-23. 10.3917/gmcc.282.0015 . hal-03577019

\section{HAL Id: hal-03577019 https://hal.sorbonne-universite.fr/hal-03577019}

Submitted on 16 Feb 2022

HAL is a multi-disciplinary open access archive for the deposit and dissemination of scientific research documents, whether they are published or not. The documents may come from teaching and research institutions in France or abroad, or from public or private research centers.
L'archive ouverte pluridisciplinaire HAL, est destinée au dépôt et à la diffusion de documents scientifiques de niveau recherche, publiés ou non, émanant des établissements d'enseignement et de recherche français ou étrangers, des laboratoires publics ou privés. 


\section{LA FRANCE ET L'EUROPE CENTRALE À VERSAILLES : OBJECTIFS STRATÉGIQUES ET COMBINAISONS DIPLOMATIQUES}

Pour les Alliés qui négocient à la Conférence de la Paix, en 1919, la stabilité de la nouvelle Europe repose sur un équilibre difficilement réalisable entre les principes de la new diplomacy et la satisfaction des exigences de sécurité. Une ambiguité apparaît rapidement dans la politique étrangère française. Elle vient du fait qu'il faut soutenir la position des États successeurs des empires effondrés au détriment des vaincus, pour des raisons essentiellement stratégiques, tout en se revendiquant du principe des nationalités. Les ambitions des négociateurs posent le problème de l'asymétrie du système international qui se met en place, la question de l'asymétrie pouvant être envisagée ici dans le sens de la tentative par la France d'accomplir une politique d'influence diplomatique, économique et militaire dans l'espace centre-oriental ${ }^{1}$. Apparait alors le problème des capacités alliées et notamment françaises à faire fonctionner un système européen basé sur des principes occidentaux, à transformer les atouts de puissance en influence politique.

\section{LES OBJECTIFS FRANÇAIS EN EUROPE CENTRALE : SÉCURITÉ ET INFLUENCE}

Dans une approche principalement géopolitique, les objectifs français impliquent la réduction de la puissance allemande, la prolongation de l'Entente, la mise en place d'une «Barrière de l'Est » composée des nouveaux alliés situés entre l'Allemagne et la Russie, les fameux « quatre piliers » (Pologne, Tchécoslovaquie, Roumanie, Yougoslavie). Cette exigence stratégique implique le soutien à une recomposition de l'Europe centrale en fonction des nationalités. On trouve les origines de cette idée dès l'année 1916, au cours de conversations entre le secrétaire-général du Quai d'Orsay, Philippe Berthelot et ses interlocuteurs Edvard Beneš et Milan Štefánik, très influents dans les cercles politiques et intellectuels français de l'époque ${ }^{2}$. Il s'agit bien sûr d'encadrer l'Allemagne, de lui trouver des contrepoids, c'est pourquoi Paris s'attache à accompagner la naissance de la Tchécoslovaquie, élément essentiel d'un système antiallemand ${ }^{3}$. De plus, le gouvernement français compte bien imprimer sa marque dans l'économie et l'industrie tchécoslovaques, en particulier à travers l'action du groupe Schneider ${ }^{4}$.

La politique française passe également par un appui à la Pologne, d'autant plus visible lors des négociations de Paris qu'il est tacitement admis parmi les Alliés que Paris doit jouer le premier rôle dans la relation avec Varsovie. Certes, David Lloyd-George et Sidney Sonnino peuvent exprimer des réserves, en particulier à propos du sort de Dantzig mais l'influence française sur les frontières occidentales du nouvel État polonais s'exerce avec l'aval des Alliés.

\footnotetext{
${ }^{1}$ Cette question de l'imposition de modèles ou d'une direction, souvent remis en cause dans les domaines politique et stratégique, est essentielle. Voir Jacques Baud, La Guerre asymétrique ou la défaite du vainqueur, Monaco, éditions du Rocher, 2005, p. 93.

${ }^{2}$ Antoine Marès, Edvard Beneš, Un drame entre Hitler et Staline, Paris, Perrin, 2015, p. 70-75 ; Bernard Michel, «Les Amitiés parisiennes de Milan Rastislav Štefánik », Bohumila Ferenčuhová (éd.), Milan Rastislav Štefánik astronome, soldat, grande figure franco-slovaque et européenne, Bratislava, Association pour l'histoire de l'Europe centrale et orientale, 1999, p. 34-40.

${ }^{3}$ Traian Sandu, «La Présence française en Europe centrale dans l'entre-deux-guerres », Revue d'Europe centrale, $\mathrm{n}^{\circ} 3,1995$, p. 147-160.

${ }^{4}$ Isabelle Davion, Mon voisin, cet ennemi. La politique de sécurité française face aux relations polonotchécoslovaques entre 1919 et 1939, Bruxelles, 2009, p. 178-189.
} 
Le gouvernement de Clemenceau a opté pour un soutien à une «Pologne forte ${ }^{5}$. La France se donne alors pour mission d'aider militairement le nouvel État polonais, en mars 1919, non seulement en envoyant en Pologne une mission militaire (tout comme elle le fait en Tchécoslovaquie) mais également en poursuivant la coopération militaire avec l'armée polonaise formée en France. Cette dernière, 1' « Armée bleue », placée sous le commandement nominal du maréchal Ferdinand Foch puisqu'il s'agit d'une armée alliée, est alors transférée en Pologne, puis en Galicie orientale, munie d'un armement et de cadres français. C'est d'ailleurs en ce début d'année 1919 que Foch explicite devant les Alliés les moyens d'utiliser les États de la «Barrière de l'Est» impliquant une action militaire de l'Entente contre la Russie révolutionnaire, en même temps qu'il soutient les visées annexionnistes de la Pologne en Galicie orientale ${ }^{6}$.

La politique française reste conditionnée par la mise en place de la «Barrière de l'Est » puis, au début du printemps 1919, par l'élaboration du « cordon sanitaire » préconisé par Foch. Elle passe par un appui aux exigences territoriales roumaines en Bessarabie, en Bucovine et en Transylvanie et par une influence militaire en Roumanie, que favorise la présence d'une partie de l'armée française d'Orient commandée par le général Henri Berthelot ${ }^{7}$. Quant au Royaume des Serbes, Croates et Slovènes, il apparaît dès sa création comme un allié de la France, ce qui pose des difficultés vis-à-vis de l'Italie, autre alliée à qui beaucoup a été promis pour favoriser son entrée en guerre, par le pacte de Londres d'avril 1915, mais qui revendique également Fiume. L'entrée des troupes serbes dans Fiume permet la création d'une base logistique francoserbe et le contrôle de voies de communication vers l'Europe centrale, au détriment de l'Italie qui redoute l'implication française dans la région ${ }^{8}$ et en vient à s'opposer aux trois autres Alliés.

Il convient d'ajouter que, même si la Barrière de l'Est est envisagée, la perspective d'un retour de la Russie dans le système international n'est pas abandonnée. Le contrepoids à l'Allemagne implique le maintien d'une Russie unifiée, c'est-à-dire sous influence occidentale dans laquelle le droit des Ukrainiens ou des Lituaniens à disposer d'eux-mêmes n'est pas forcément respecté ${ }^{9}$. Cette politique est d'ailleurs appliquée sur le terrain par les militaires français en Ukraine ${ }^{10}$.

La question essentielle demeure celle de la neutralisation de l'Allemagne. Pour Paris, la sécurité du territoire passe par le contrôle de la réduction de la puissance allemande, donc

\footnotetext{
${ }^{5}$ Jadwiga Wala, «Une Pologne forte dans la nouvelle Europe vue par la France : unité nationale et protection des minorités », Isabelle Davion, Jerzy Kłoczowski, Georges-Henri Soutou (éd.), La Pologne et l'Europe du partage à l'élargissement (XVIII $-X X I^{e}$ siècles), Paris, PUPS, 2007, p. 111-118.

${ }^{6}$ Vincennes, Service historique de la Défense-Département Terre (SHD-Terre), 4N93, EMA, dossier 1, Procèsverbal, séance du Conseil supérieur de la guerre, 17 mars 1919 ; Frédéric Dessberg, «La Pensée et les projets stratégiques du maréchal Foch en Europe centrale et orientale (1919-1929) », Rémy Porte, François Cochet (éd.), Ferdinand Foch (1851-1929). Apprenez à penser, Paris, Sotéca, 2010, p. 311-332.

${ }^{7}$ Alexandru Ocsa, «La Politique de la France et de l'Italie au sud-est de l'Europe pendant les premières années après la Première Guerre mondiale », Revue internationale d'histoire militaire, $\mathrm{n}^{\circ}$ 83, 2003, p. 59-71.

${ }^{8}$ Ministère des Affaires étrangères français (MAEF), Documents diplomatiques français (DDF), Armistices et Paix (27 septembre 1918-17 janvier 1919), vol. I, Bruxelles, PIE Peter Lang, document $\mathrm{n}^{\circ}$ 297, télégramme de Stephen Pichon (ministre des Affaires étrangères) aux ambassadeurs Paul Cambon (Londres), Jules Jusserand (Washington) et Camille Barrère (Rome), 5 décembre 1918.

9 Ainsi, une convention franco-britannique est signée le 23 décembre 1917, instaurant un partage de zones d'influence dans l'ancien empire russe. Voir Mariya Romanova, La Politique étrangère française et l'Ukraine de la fin de la Première Guerre mondiale à 1921, thèse de doctorat, université Paris-Sorbonne, juin 2016, p. 128132 ; Anne Hogenhuis-Seliverstoff, Les Relations franco-soviétiques, 1917-1924, Paris, Publications de la Sorbonne, 1981, p. 56.

${ }^{10}$ Vincennes, SHD-Terre, 16N3021, Groupe de l'Avant, dossier $n^{\circ} 41$, télégramme de Ferdinand Foch au général Henri Niessel (Mission militaire française en Russie), 25 novembre 1917.
} 
notamment par l'interdiction de l'annexion de l'Autriche par l'Allemagne. Les deux problèmes sont liés. En dépit de l'obsession allemande de la France, même si l'Autriche, c'est « ce qui reste », selon la formule prêtée à Clemenceau, rappelons que lorsque le premier jour de première réunion du Conseil des Quatre, Wilson énumère les sujets urgents et les plus difficiles à régler : les réparations, la sécurité de la France, la frontière italienne en Adriatique, Clemenceau ajoute le sort de "l'Autriche allemande ${ }^{11}$. Il s'agit donc de faire en sorte que l'Autriche soit viable, qu'elle ne soit pas attirée par l'Allemagne et que, pour cela, soit créé un espace de solidarité dans le cadre d'une confédération danubienne.

Mais ce projet se heurte à l'opposition italienne qui redoute qu'une confédération soit le premier pas vers une restauration Habsbourg dangereuse pour 1'Italie. Français et Italiens trouveront un compromis sous la forme de la «garantie mutuelle »: ni Anschluss, ni Confédération danubienne ${ }^{12}$. La question de l'Autriche, bien que secondaire pour la France, intéresse donc au plus haut point la sécurité française. C'est tout $l^{\prime}$ 'enjeu de la querelle francoitalienne liée à la question adriatique. L'Italie rappelle les promesses qui lui ont été faites en avril 1915 mais l'allié serbe, fondateur de la Yougoslavie, a également ses revendications. La construction de la Barrière de l'Est est donc troublée par les considérations diplomatiques. Laisser l'Italie étendre son influence dans les Balkans, c'est lui donner l'accès à l'Europe danubienne et aux voies de communications. Or, la France veut contrôler les accès ferroviaires vers l'Europe centrale pour assurer le ravitaillement de ses alliés. D'un autre côté, satisfaire les revendications yougoslaves, c'est se fâcher avec un allié. Dans cette question aussi, les considérations stratégiques l'emportent sur le droit des peuples à l'autodétermination qui se trouve donc être très malléable dans l'esprit des dirigeants français.

\section{LA POSITION FRANÇAISE : ENTRE DROIT À L'AUTODÉTERMINATION ET IMPERATIFS STRATÉGIQUES}

Proclamée à la fois par Wilson et Lénine, la notion de droit des peuples à disposer d'euxmêmes n'est encore, au moment des négociations de Paris, qu'un principe politique, socle essentiel d'une paix juste. Clemenceau ne l'envisage que dans le cadre des réalités géopolitiques du moment ${ }^{13}$. La France a mis en avant de manière récurrente le soutien aux nationalités au cours du XIX ${ }^{\mathrm{e}}$ siècle, avant de le mettre en sourdine dans les années 1890, à la suite de l'alliance franco-russe et pendant la Grande Guerre. Le principe n'est en effet plus envisageable, appliqué à l'Empire russe, notamment en ce qui concerne la Pologne, mais aussi à propos de l'Ukraine et des pays baltes ${ }^{14}$. Il est véritablement adopté quand le Conseil des ministres approuve les dispositions du Congrès sur les nationalités opprimées de l'empire d'Autriche-Hongrie qui se tient à Rome en avril 1918 et surtout, deux mois plus tard, par une déclaration solennelle des Alliés qui appellent notamment à la création d'un État polonais ${ }^{15}$.

Or, le principe du droit des peuples à disposer d'eux-mêmes connaît finalement de nombreuses entorses. En cas d'arbitrage sur les questions frontalières, les décisions sont

\footnotetext{
${ }^{11}$ Paul Mantoux, La Délibération du Conseil des Quatre (24 mars-28 juin 1919), t. 1, jusqu'à la remise à la délégation allemande des conditions de paix, Paris, Éditions du CNRS, 1955, 24 mars 1919, p. 13.

12 Anne-Sophie Nardelli-Malgrand, «Sécurité et reconstruction. L’Anschluss au cœur des relations francoitaliennes de 1919 à $1922 », M E F R I M$, vol. 2, nº120, 2008, p. 281-296.

${ }^{13}$ Dzovinar Kévonian, «Refondation des relations internationales : Clemenceau et le droit des peuples à disposer d'eux-mêmes », Sylvie Brodziak, Caroline Fontaine (éd.), Clemenceau et la Grande Guerre, 1906-1929, La Crèche, Geste éditions, p.183-202.

${ }^{14}$ Georges-Henri Soutou (éd.), Recherches sur la France et le problème des nationalités pendant la Première Guerre mondiale (Pologne, Ukraine, Lituanie), Paris, Presses de l'université de Paris-Sorbonne, 1995.

15 Jean-Baptiste Duroselle, Clemenceau, Fayard, 1988, p. 812 ; Georges-Henri Soutou, La Grande Illusion ..., op. cit., p. 270.
} 
généralement prises au bénéfice des États alliés (Pologne, Tchécoslovaquie, Royaume des Serbes, des Croates et des Slovènes, Roumanie agrandie). Lors de la Conférence de la Paix, Clemenceau soutient par exemple le projet de Grande Pologne de Roman Dmowski qui prévoit d'inclure des populations non-polonaises (Ukrainiens, Lituaniens, par exemple) dans le nouvel État. Il plaide pour l'attribution à la Pologne non seulement d'un accès à la mer Baltique mais également du corridor longeant la Vistule. Sur le terrain, la commission de délimitation des frontières, présidée par le général Charles Dupont, fonctionne ensuite dans l'unique objectif d'appliquer strictement le traité de Versailles, ce qui s'avère particulièrement difficile ${ }^{16}$. Il apparaît donc que les considérations stratégiques l'emportent sur le principe du droit des peuples à disposer d'eux-mêmes : «Il faut accepter des accrocs inévitables au principe du droit des peuples à disposer d'eux-mêmes si nous voulons garder ce principe même » déclare Clemenceau le 27 mars 1919 en s'opposant à Lloyd-George qui, lui, réfute les arguments stratégiques dans la question de Dantzig ${ }^{17}$.

Le principe de l'autodétermination est particulièrement difficile à appliquer dans le cas où un différend territorial survient entre deux composantes de la Barrière de l'Est. Le cas se présente dans la région de Teschen, en Silésie, riche région minière convoitée à la fois par la Pologne et la Tchécoslovaquie. Dans ce cas encore, les considérations stratégiques l'emportent. La Tchécoslovaquie emporte la mise à la suite d'un partage avantageux, les Polonais se trouvant alors, à la fin de juillet 1920, en très mauvaise posture face à la Russie bolchevique. Il est de plus indispensable pour les Français que leur allié tchécoslovaque, considéré comme le plus proche du gouvernement français et le plus viable en Europe centrale, puisse disposer d'un accès garanti au charbon de Teschen ${ }^{18}$.

Les arguments stratégiques l'emportent généralement dans la fixation des frontières en Europe centrale. C'est par exemple le cas en ce qui concerne la frontière roumano-hongroise, en Transylvanie ${ }^{19}$. Pour les Français, un enjeu essentiel est de donner les moyens à la Pologne d'être ravitaillée en matériel de guerre. Les Italiens ont un point de vue différent, lié à leur situation à Fiume. La question qui anime les débats entre Alliés est de décider si la population des villes doit être uniquement prise en compte ou si les habitants du voisinage des villes sont également concernés. Dans cette affaire, les Italiens prennent position pour la population hongroise, principalement urbaine, contre les Roumains peuplant surtout les campagnes, de la même manière qu'ils revendiquent Fiume, ville qu'ils considèrent comme italienne et entourée de populations croates et slovènes ${ }^{20}$. À l'inverse, Paris favorise l'allié roumain contre les revendications de l'ancien ennemi hongrois. La question de la Hongrie, réglée par le traité de Trianon, montre le mieux comment les principes annoncés à la Conférence de la paix se réduisent face aux impératifs stratégiques et au contexte politique européen particulièrement chaotique de l'année 1919. D'ailleurs, les décisions prises sur le terrain, notamment par le général Berthelot et le maréchal Foch, l'emportent sur les principes au moment de tracer la frontière roumano-hongroise. Les Alliés de la France doivent être viables ${ }^{21}$.

La France accompagne un nouveau type de construction aux États successeurs des empires : le modèle de l'État-nation s'impose mais à l'intérieur des États, le problème des

\footnotetext{
${ }^{16}$ Vincennes, SHD-Terre, 4N81, rapport du général Dupont à l'EMA, 28 mai 1920.

${ }^{17}$ Paul Mantoux, Les Délibérations du Conseil des Quatre..., op. cit., 27 mars 1919, p. 44.

${ }^{18}$ Isabelle Davion, Mon voisin, cet ennemi. La politique de sécurité française face aux relations polonotchécoslovaques entre 1919 et 1939, Bruxelles, PIE Peter Lang, 2009, p.133-134.

19 Traian Sandu, Le Système de sécurité français en Europe centre-orientale. L'exemple roumain, Paris, L'Harmattan, 1999, p. 55-72.

${ }^{20}$ Francesco Caccamo, L'Italia e la « Nuova Europa ». Il confronto sull'Europa orientale alla conferenza di Parigi (1919-1920), Milano, Luni Editrice, 2000, p. 67.

${ }^{21}$ Dzovinar Kévonian, « Refondation des relations internationales... », op. cit., p. 201.
} 
nationalités se pose tout autant que dans les empires car il n'a pas été possible, ni même souhaitable, de faire correspondre un État à chaque nationalité : la Roumanie contient des minorités hongroise, allemande, ukrainienne, bulgare, russe ; la Pologne contient des minorités ukrainienne, biélorusse, allemande, etc. Force est donc de reconnaître qu'une nationalité peut primer sur toutes les autres à l'intérieur d'un même État. Certaines nationalités ont obtenu une reconnaissance de la part des Alliés, conformément au principe de l'autodétermination mais la France est souvent conduite à agir en toute ambiguïté. La Pologne doit ainsi rester dans ses limites ethnographiques mais Paris soutient le corridor sur la Vistule, ainsi que la prise de la Galicie orientale, au détriment de la population ukrainienne, minimisant à cette occasion les violences commises à Lemberg par les troupes polonaises ${ }^{22}$.

Le principe d'autodétermination se trouve donc face à de grandes difficultés d'adaptation en Europe centrale car pour les grandes puissances, en particulier pour la France, il demeure conditionné aux intérêts stratégiques. Les entorses au principe sont acceptées parce que les dirigeants sont pragmatiques et que la paix doit nécessairement être le fruit d'un compromis. Parmi les exigences de la Conférence figure la viabilité des États, en lien avec l'établissement des frontières. Il s'agit également de conserver une unité au moins apparentes entre les principaux Alliés, en même temps que les revendications majeures des Alliés d'Europe centrale doivent être satisfaite, ce qui peut produire des tensions, comme nous l'avons vu dans le contentieux opposant les Italiens et les Yougoslaves.

\section{FRONTIÈRES ET ALLIANCES EN EUROPE CENTRALE}

Les objectifs français lors de la Conférence de la paix visent à une projection de l'influence française en Europe centrale, où Paris espère remplacer l'influence allemande grâce à un nouveau réseau d'alliances tourné vers la France mais établissant également des liens entre les États successeurs des Empires effondrés. Immanquablement, les autorités françaises peuvent avoir tendance à privilégier les intérêts des alliés de l'Est que l'on espère fidèles et liés aux intérêts français, plutôt que ceux des anciens ennemis vaincus. En 1919, le contexte de désordre généralisé de l'Europe centrale, les contentieux frontaliers et les luttes politiques internes compliquent le jeu français. Il arrive alors fréquemment que, loin des négociations de Paris, la politique des faits accomplis prime dans la région et oriente donc les décisions des Alliés. Les Quatre Grands, à l'exception notable de l'Italie, prennent ainsi le parti d'États alliés lancés dans des actions militaires. Dans ce cas, la lutte contre la propagation révolutionnaire est un argument qui pèse. C'est pourquoi la création de la République des Conseils de Hongrie marque un tournant important. Elle pousse à une plus grande rigueur par crainte d'une collusion germano-russe mais surtout pas crainte de voir naître une alliance objective entre le bolchevisme et le nationalisme des pays vaincus. Rappelons qu' au Conseil des Quatre, les alliés britannique et italien ne se privent pas de faire porter la responsabilité de la révolution en Hongrie sur l'établissement par les Alliés eux-mêmes d'une ligne de démarcation considérée comme future frontière et par la rigidité des chefs de l'armée de Hongrie ${ }^{23}$. Dans le contexte de grande menace révolutionnaire, l'influence de Foch est très importante. La révolution et la possibilité d'une alliance avec l'Allemagne le poussent à élaborer une série de plans dans les premiers mois de 1919. Il s'agit d'utiliser un corps expéditionnaire allié à travers la Pologne,

\footnotetext{
${ }^{22}$ Paul Mantoux, Les Délibérations du Conseil des Quatre, (24 mars-28 juin 1919), t. 2, jusqu'à la remise à la délégation allemande des conditions de paix, Paris, Éditions du CNRS, 1955, p. 150-155, 21 mai 1919 ; GeorgesHenri. Soutou, La Grande Illusion. Quand la France perdait la paix, 1914-1920, Paris, Tallandier, 2015, p. 265. ${ }^{23}$ Paul Mantoux, Les Délibérations du Conseil des Quatre, t. 1, op. cit., p. 99.
} 
d'utiliser cette dernière et la Roumanie comme « la plus solide barrière contre le bolchevisme triomphant en Russie ${ }^{24}$ » puis, en mode dégradé, de se contenter d'un « cordon sanitaire ».

Dans les États eux-mêmes, enfin, l'influence française peine parfois à s'installer. La France parvient à prendre le contrôle d'entreprises mais pas forcément à financer l'influence française à l'est. C'est «l'impérialisme du pauvre» décrit par Georges-Henri Soutou ${ }^{25}$. Le modèle militaire français réussit toutefois à s'imposer en Tchécoslovaquie, où la Mission militaire française, commandée par le général Maurice Pellé, réussit à supplanter la Mission militaire italienne du général Luigi Piccione, le général français devenant même le chef d'étatmajor de l'armée tchécoslovaque ${ }^{26}$. En Roumanie, l'influence militaire française, avec la présence du général Berthelot, reste exclusive, au moins dans les années 1920. En Pologne, la France a pour ambition d'exercer un monopole. Cet objectif est annoncé de manière très explicite dans la correspondance du chef de la Mission militaire, le général Paul Prosper Henrys, pour qui l'instruction de militaires polonais par du personnel français doit permettre à l'influence française « de rayonner sur tout le territoire polonais et d'atteindre la généralité de l'armée polonaise ${ }^{27} »$.

Le prestige de l'armée française à l'issue de la guerre et le soutien apporté par Paris aux Alliés de l'Est n'épargnent cependant pas aux Français d'affronter des difficultés face à un gouvernement polonais déterminé à ne pas trop dépendre des Français. La Mission militaire française en Pologne réussit tant bien que mal à écarter, pour un temps, la concurrence étrangère dans la vente d'armes, les officiers français sont présents dans les centres d'instruction, contribuent à la création d'une École de Guerre, conseillent les états-majors durant la guerre de 1920. Contre cette influence s'impose la volonté du chef de l'État, Józef Piłsudski, de refuser toute implication française trop forte. De fait, 1' «Armée bleue » formée en France et comportant des cadres et des combattants français ne s'impose pas comme modèle pour l'armée polonaise $^{28}$.

Les attentes des alliés de l'Est, en réalité, ne sont pas entièrement comblées. Ils en viennent à se demander si, dans les moments cruciaux, la France est capable d'intervenir efficacement sur le plan militaire et diplomatique. Par exemple, le soutien de Paris à la Pologne dans la question de la Haute Silésie n'a pas débouché sur l'attribution de la totalité de la région à Varsovie. En ce qui concerne Prague, la guerre en Slovaquie a montré les limites de l'engagement militaire français. Du reste, les pays d'Europe centrale mènent leur propre politique dans la région. La Tchécoslovaquie, par exemple, cherche à exercer sa propre influence vis-à-vis de l'Autriche, en conduisant une politique de rapprochement avec l'Italie lorsque la France se rapproche de la Hongrie, en 1920. Edvard Beneš, logiquement, agit en fonction des intérêts de la Tchécoslovaquie davantage qu'en référence à un lien d'alliance. On le voit, l'influence française est remise en question chez ses alliés et le mouvement ne fera que s'amplifier au fil des années.

\footnotetext{
${ }^{24}$ Vincennes, SHD-Terre, 4N93, dossier $\mathrm{n}^{\circ} 1,17$ mars 1919.

${ }^{25}$ Georges-Henri Soutou, «L'impérialisme du pauvre : la politique économique du gouvernement français en Europe centrale et orientale de 1918 à 1929 », Relations internationales, n 7, automne 1976, p. 219-239.

${ }^{26}$ Antoine Marès, « Mission militaire et relations internationales : l'exemple franco-tchécoslovaque, 1918-1925 », Revue d'histoire moderne et contemporaine, $\mathrm{n}^{\circ} 4,1983$, p. 559-586.

${ }^{27}$ DDF 1920, t. 1, Paris, Imprimerie nationale, 1997, note du général Henrys au ministre de la Guerre et au $2^{\mathrm{e}}$ Bureau de l'état-major, 5 mars 1920.

${ }^{28}$ Lech Maliszewski, «Louis Faury (1874-1947) : entre gloire et oubli », Revue historique des armées, $\mathrm{n}^{\circ} 260$, 2010, p. 37-44.
} 


\section{CONCLUSION}

La période de la négociation des traités de paix montre l'asymétrie du système qui se met en place. On assiste à une hiérarchisation des relations internationales du point de vue de la France qui réfléchit en terme d'influence, voire d'hégémonie et adapte sa diplomatie en fonction de l'importance donnée au partenaire, qu'il s'agisse de l'allié, par exemple britannique, qu'il faut ménager, ou du partenaire allié ou ennemi en Europe centrale et orientale, que l'on cherche à influencer de manières diverses. Mais on constate surtout une grande difficulté pour la France à dicter de manière unilatérale les termes de la relation. Les faiblesses du système de sécurité français sont évidentes : manque de moyens d'influence financiers mais aussi militaires face aux concurrents étrangers, intérêts nationaux des États qui rechignent à former le bloc homogène souhaité par la France. De plus, les partenaires restent attachés à leurs droits, font référence à des identités, à des légitimités historiques, territoriales, à une aspiration à l'autonomie. Ils mettent ainsi en cause l'influence qui cherche à s'étendre. Le jeu diplomatique, ensuite, vient perturber la mise en place du système européen, en même temps que le contexte international très troublé de l'époque. 